CERN-TH/2002-312

hep-lat/0211004

\title{
Comparison of $\mathrm{SO}(3)$ and $\mathrm{SU}(2)$ Lattice Gauge Theory
}

\author{
Philippe de Forcrand ${ }^{a, b, 1}$ and Oliver Jahn ${ }^{a, 2}$ \\ ${ }^{a}$ Institute for Theoretical Physics, ETH Zürich, CH-8093 Zürich, Switzerland \\ ${ }^{b}$ CERN, Theory Division, CH-1211 Genève 23, Switzerland
}

\begin{abstract}
The Villain form of $\mathrm{SO}(3)$ lattice gauge theory is studied and compared to Wilson's $\mathrm{SU}(2)$ theory. The topological invariants in $\mathrm{SO}(3)$ which correspond to twisted boundary conditions in $\mathrm{SU}(2)$ are discussed and lattice observables are introduced for them. An apparent $\mathrm{SO}(3)$ phase with negative adjoint Polyakov loop is explained in terms of these observables. The electric twist free energy, an order parameter for the confinement-deconfinement transition, is measured in both theories to calibrate the temperature. The results indicate that lattices with about $700^{4}$ sites or larger will be needed to study the $\mathrm{SO}(3)$ confined phase. Alternative actions are discussed and an analytic path connecting $\mathrm{SO}(3)$ and $\mathrm{SU}(2)$ lattice gauge theory at weak coupling is exhibited. The relevance for confinement of the centre of the gauge group is discussed.
\end{abstract}

\footnotetext{
${ }^{1}$ forcrand@phys.ethz.ch

${ }^{2}$ jahn@phys.ethz.ch
} 


\section{Motivation}

Although SO(3) lattice gauge theories (LGT) are expected to have the same continuum limit as theories with gauge group $\mathrm{SU}(2)$, they are interesting for at least two reasons. First, the equivalence of the non-perturbative continuum limits has not been proven yet; there are in fact some indications that they might be different. Second, they provide a tool to study the role of the centre of the gauge group, which is often considered to be closely related to confinement: the centre of $\mathrm{SO}(3)$, as opposed to that of $\mathrm{SU}(2)$, is trivial.

The expectations on the continuum limit are largely based on the naive continuum limit as obtained by expanding the classical action around its minima. As $\mathrm{SO}(3) \cong \mathrm{SU}(2) / \mathbb{Z}_{2}$ is locally indistinguishable from $\mathrm{SU}(2)$, in particular in any small region around the identity, plaquette actions based on $\mathrm{SO}(3)$ and $\mathrm{SU}(2)$ link variables have the same naive continuum limit. Invoking standard arguments of universality of the continuum limit, one expects the non-perturbative continuum limit to be the same, too. However, this expectation is not based on a rigorous theorem and should be checked for each individual case. Studies of the Stefan-Boltzmann law in both theories for instance have raised doubts [1].

Studies of mixed actions containing both $\mathrm{SU}(2)$ and $\mathrm{SO}(3)$ parts [the latter being considered as the adjoint representation of $\mathrm{SU}(2)$ ] have not yet been able to demonstrate this universality. Rather they reveal a line of first order phase transitions separating the weak coupling regions of the pure $\mathrm{SO}(3)$ and $\mathrm{SU}(2)$ theories [2, 3, so the continuum limits are not analytically connected in this plane of couplings. The location of the phase transitions is essentially independent of the lattice size, so they are considered as bulk. In the pure $\mathrm{SO}(3)$ theory, the transition occurs at very weak coupling. This puts an upper bound on the lattice spacing in the weak coupling phase, and prohibitively large lattices are required to study low temperatures.

A further peculiarity of the $\mathrm{SO}(3)$ theory is the occurance of a new meta-stable "phase" (long-lived state in the Monte-Carlo simulation) with a negative expectation value of the adjoint Polyakov loop at weak coupling [4, 5. Apart from the Polyakov loop, this "phase" seems to be very similar to the standard phase with positive adjoint Polyakov loop. Still its existence has fuelled speculations about differences between $\mathrm{SO}(3)$ and $\mathrm{SU}(2)$ theories. We will show that the mysterious phase can be understood in terms of the $\mathrm{SU}(2)$ theory.

The question of whether $\mathrm{SO}(3)$ and $\mathrm{SU}(2)$ LGT describe the same quantum theory is also important for our understanding of the role of the centre of the gauge group. A common belief asserts that deconfinement is caused by the breakdown of the centre symmetry [6]. However, it has been argued that this symmetry is a peculiarity of the lattice theory and not present in other regularisations [7]. In the $\mathrm{SO}(3)$ lattice theory, the centre symmetry is absent and cannot be broken. If this theory has a confinement-deconfinement transition, too, it has to be explained by a different mechanism.

While the centre symmetry is absent, it should be noted that centre vortices do exist in an $\mathrm{SO}(3)$ theory: their definition actually does not rely on the centre but on 
a non-trivial first homotopy group of the gauge group modulo its centre. As $S O(3)$ is $S U(2)$ modulo its centre $\mathbb{Z}_{2}$, this is the same for both groups, $\pi_{1}\left[\mathrm{SU}(2) / \mathbb{Z}_{2}\right]=$ $\pi_{1}[\mathrm{SO}(3)]=\mathbb{Z}_{2}$. So confinement mechanisms based on centre vortices can apply to both theories equally well.

In the absence of the centre symmetry, the question of an order parameter for the confinement-deconfinement mechanism arises. The Polyakov loop in the fundamental representation is useless because its sign is not determined. The adjoint Polyakov loop is not good either, since it has a non-zero expectation value also in the confined phase because adjoint charges can be screened by gluons. A useful alternative is known for the $\mathrm{SU}(2)$ theory. The expectation value of a large spatial 't Hooft loop follows a perimeter law in the confined phase and an area law in the deconfined phase, the coefficient defining the "dual string tension" [8, 9]. A particularly convenient way to measure 't Hooft loops is provided by twisted boundary conditions on a hypercubic lattice (a torus). These introduce chromo-electric and -magnetic fluxes through the torus and correspond to 't Hooft loops of maximal size [10]. Their expectation value has been measured both at zero [11] and at finite temperatures [12, 13], and perimeter respectively area law have been demonstrated. We will show that the free energy of these fluxes can also be measured in an $\mathrm{SO}(3)$ LGT. As 't Hooft loops can be thought of as generating centre vortices, this shows that the latter do indeed play a role. Preliminary accounts of the present work have been given in [14, 15].

We begin in Sec. 2 with a review of the topological invariants of $\mathrm{SU}(N)$ and $\mathrm{SU}(N) / \mathbb{Z}_{N}$ lattice gauge theory on a 4 -torus, and of the implementation of twisted boundary conditions in $\mathrm{SU}(N)$ LGT. Section 3 recalls a formulation of the $\mathrm{SU}(2)$ theory as a coupled $\mathrm{SO}(3)-\mathbb{Z}_{2}$ theory. This formulation motivates a definition of twist in the $\mathrm{SO}(3)$ theory which is presented in Sec. 4. In Sec. 5, an interpretation of the "phase" with negative adjoint Polyakov loop is presented. Section [6] describes our numerical computation of the free energy of electric twist in $\mathrm{SO}(3)$. Before the conclusions, a discussion of alternative actions and an analytic path between $\mathrm{SO}(3)$ and $\mathrm{SU}(2)$ at weak coupling are proposed. The general relation between Polyakov loops and twist is reviewed in the appendix.

\section{Topology in $\mathrm{SU}(N) / \mathbb{Z}_{N}$ and $\mathrm{SU}(N)$ on the torus}

In the continuum, there is no local difference between $\mathrm{SU}(N)$ and $\mathrm{SU}(N) / \mathbb{Z}_{N}$ gauge fields. The gauge potential can be expressed either in the fundamental representation (with generators $t^{a}$ ) or in the adjoint one (with generators $T^{a}$ ),

$$
A_{\mu}^{\mathrm{F}}=A_{\mu}^{a} t^{a} \quad \text { or } \quad A_{\mu}^{\mathrm{A}}=A_{\mu}^{a} T^{a} .
$$

The components $A_{\mu}^{a}$ are always the same. The only difference is in the boundary conditions, since the transition functions $\Omega_{\mu}$ take values in the gauge group. On a torus with extent $L_{\nu}$ in direction $e_{\nu}$ :

$$
A_{\mu}^{\mathrm{F} / \mathrm{A}}\left(x+L_{\nu} e_{\nu}\right)=\Omega_{\nu}^{\mathrm{F} / \mathrm{A}}(x)\left(A_{\mu}^{\mathrm{F} / \mathrm{A}}(x)-\mathrm{i} \partial_{\mu}\right) \Omega_{\nu}^{\mathrm{F} / \mathrm{A} \dagger}(x) .
$$


The transition functions satisfy the consistency conditions

$$
\begin{aligned}
& \Omega_{\mu}^{\mathrm{F}}\left(x+L_{\nu} e_{\nu}\right) \Omega_{\nu}^{\mathrm{F}}(x)=z_{\mu \nu} \Omega_{\nu}^{\mathrm{F}}\left(x+L_{\mu} e_{\mu}\right) \Omega_{\mu}^{\mathrm{F}}(x) \\
& \Omega_{\mu}^{\mathrm{A}}\left(x+L_{\nu} e_{\nu}\right) \Omega_{\nu}^{\mathrm{A}}(x)=\Omega_{\nu}^{\mathrm{A}}\left(x+L_{\mu} e_{\mu}\right) \Omega_{\mu}^{\mathrm{A}}(x) .
\end{aligned}
$$

The twist $z_{\mu \nu}=\exp \left(\frac{2 \pi \mathrm{i}}{N} n_{\mu \nu}\right)$ with integer $n_{\mu \nu}$ has to be an element of the centre $\mathbb{Z}_{N}$ of $\mathrm{SU}(N)$. The group $\mathrm{SU}(N) / \mathbb{Z}_{N}$ has trivial centre, so no twist is allowed for $\Omega_{\mu}^{\mathrm{A}}$. With (3) and (4), both descriptions are equivalent also globally, as any set of transition functions in $\mathrm{SU}(N) / \mathbb{Z}_{N}$ can be lifted to $\mathrm{SU}(N)$ and any in $\mathrm{SU}(N)$ can be projected to $\mathrm{SU}(N) / \mathbb{Z}_{N}$. The twist $z=\left\{z_{\mu \nu}\right\}$, specified by the consistency conditions in the case of $\mathrm{SU}(N)$ fields, becomes an ordinary topological invariant like the instanton number for $\mathrm{SU}(N) / \mathbb{Z}_{N}$ fields. In a slight abuse of language, we shall call it twist also there. In addition to the 6 twists $z_{\mu \nu} \in \pi_{1}\left[\mathrm{SU}(N) / \mathbb{Z}_{N}\right]=\mathbb{Z}_{N}$ which are associated with two-dimensional sections of the torus ( $\mu \nu$-planes), there is one more topological invariant $q \in \pi_{3}\left[\mathrm{SU}(N) / \mathbb{Z}_{N}\right]=\mathbb{Z}$ associated with the bulk [16, 17. The usual topological charge (instanton number) $Q$ is a combination of both,

$$
Q=q-\frac{\kappa}{N} \quad \text { with } \quad \kappa=\frac{1}{8} \varepsilon_{\mu \nu \rho \sigma} n_{\mu \nu} n_{\rho \sigma} .
$$

It can take fractional values for non-zero $n$.

On a lattice, periodic link variables represent all possible boundary conditions with trivial consistency conditions [i.e. (44) or (3) with $z_{\mu \nu}=1$ ]. Therefore, the $\mathrm{SU}(N) / \mathbb{Z}_{N}$ lattice theory (with periodic boundary conditions) automatically contains all "twist" and instanton sectors while the periodic $\mathrm{SU}(N)$ theory contains all instanton sectors but only trivial twist. A non-trivial (fixed) twist $z$ can be implemented by modifying the periodic boundary conditions to $U_{\nu}\left(x+L_{\mu}\right)=z_{\mu \nu} U_{\nu}(x)$ for $\mu<\nu$ for a single value of $x_{\mu}$. Alternatively, one can work with periodic link variables by absorbing the factor $z_{\mu \nu}$ into one plaquette in each $\mu \nu$-plane [18. The partition function of $\mathrm{SU}(N)$ LGT with Wilson action for twist $z$ then becomes

$$
Z_{\mathrm{SU}(N)}^{\mathrm{Wilson}}(z)=\int_{\mathrm{SU}(N)} \prod_{l} \mathrm{~d} U_{l} \exp \left[-\beta \sum_{p}\left(1-\frac{1}{N} \operatorname{Re}\left(\zeta_{p} \operatorname{Tr}_{\mathrm{F}} U_{p}\right)\right)\right]
$$

where $l$ labels links, $p$ plaquettes and

$$
\zeta_{x, \mu \nu}= \begin{cases}z_{\mu \nu} & \text { if } x_{\mu}=x_{\nu}=0 \\ 1 & \text { otherwise }\end{cases}
$$

In the $\mathrm{SU}(N) / \mathbb{Z}_{N}$ lattice theory, the twist cannot be imposed in this way by the boundary conditions - all twists $z$ are included within periodic boundary conditions. A "field-theoretic" definition is not possible either, since $z$ does not have a local representation as an integral like the topological charge $\left(Q \propto \int F \tilde{F}\right){ }^{3}$ However, an

\footnotetext{
${ }^{3} \mathrm{In}$ terms of $\mathrm{U}(N)$ fields they do [19], but we will not pursue this any further.
} 
$\mathrm{SU}(N) / \mathbb{Z}_{N}$ lattice theory can also be described redundantly in terms of $\mathrm{SU}(N)$ variables. Just imagine mapping each link variable to an $\mathrm{SU}(N)$ element, with arbitrary $\mathbb{Z}_{N}$ phase. The resulting link configuration will in general not be periodic, but only periodic modulo $\mathbb{Z}_{N}$. The transition functions will satisfy twisted consistency conditions like (3), but the twist will be a priori arbitrary. One way of assigning a unique value, would be some smoothness requirement. One could, for instance, try to fix the ambiguity by demanding that the $\mathrm{SU}(N)$ plaquette variables be as close to $\mathbb{1}$ as possible. This is not natural, however, since $\mathrm{SU}(N) / \mathbb{Z}_{N}$ lattice gauge fields describe continuum gauge fields with $\mathbb{Z}_{N}$ monopoles. Attempting a "smooth" lift to $\mathrm{SU}(N)$ ignores the latter. We therefore choose a different approach, defining twist by analogy with the $\mathrm{SU}(N)$ theory in a formulation in $\mathrm{SU}(N) / \mathbb{Z}_{N}$ and $\mathbb{Z}_{N}$ variables.

\section{$3 \quad \mathrm{SU}(2)$ LGT as a coupled $\mathrm{SO}(3)-\mathbb{Z}_{2}$ theory}

In order to carry the definition of twist over to an $\mathrm{SU}(N) / \mathbb{Z}_{N} \mathrm{LGT}$, we invoke a formulation of the $\mathrm{SU}(N)$ theory in terms of $\mathrm{SU}(N) / \mathbb{Z}_{N}$ and $\mathbb{Z}_{N}$ variables. We specialise to $N=2$, the case studied numerically in this paper.

The Wilson partition function for twist $z$ (6) can be expressed as [20, 21, 22, 23 .

$$
Z_{\mathrm{SU}(2)}^{\mathrm{Wilson}}(z)=\mathcal{N} \sum_{\alpha_{p}= \pm 1} \int \prod_{l} \mathrm{~d} U_{l} \exp \left(\frac{\beta}{2} \sum_{p} \alpha_{p} \operatorname{Tr}_{\mathrm{F}} U_{p}\right) \prod_{c} \delta\left(\alpha_{c}, 1\right) \prod_{\mu<\nu} \delta\left(\alpha_{\mu \nu}, z_{\mu \nu}\right)
$$

where $c$ labels cubes and

$$
\begin{aligned}
\alpha_{c} & =\prod_{p \in \partial c} \alpha_{p}, \\
\alpha_{\mu \nu} & =\prod_{x_{\mu}, x_{\nu}} \alpha_{x, \mu \nu}
\end{aligned}
$$

with $x_{\rho}$ fixed for $\rho \neq \mu, \nu$ in the last equation. The factor $\mathcal{N}$ represents a change of normalisation. The choice of $\mathbb{Z}_{2}$ plaquette variables here is different from that of [21, 22]: they are related as $\alpha_{p}=\sigma_{p} \operatorname{sgn} \operatorname{Tr} U_{p}$.

Equation (81) can be understood as follows. The first set of constraints (on $\alpha_{c}$ ) can be interpreted as the Bianchi identity for the field strength $\alpha_{p}$ of a $\mathbb{Z}_{2}$ gauge theory. It forbids monopoles and ensures local integrability: $\alpha_{p}$ can be obtained from a $\mathbb{Z}_{2}$ link field (potential) $\gamma_{l}$,

$$
\alpha_{p}=\prod_{l \in \partial p} \gamma_{l} \quad \text { (locally) }
$$

This is possible whenever the product of $\alpha_{p}$ over all closed surfaces is 1 . In $\mathbb{R}^{4}$, all closed surfaces can be constructed from elementary cubes, so the first set of constraints suffices. On a torus, there are 6 independent winding surfaces which 
cannot be obtained in this way. The second set of constraints fixes the products of $\alpha_{p}$ on these. If $z_{\mu \nu}=1, \alpha_{p}$ can then be integrated globally. Otherwise, one has to make $\alpha_{\mu \nu}$ trivial first. This is achieved by dividing $\alpha_{p}$ by any representative with the same $\alpha_{\mu \nu}$, for instance (77); so the potential is introduced as

$$
\alpha_{p}=\zeta_{p} \prod_{l \in \partial p} \gamma_{l}
$$

With this representation, the sum over $\alpha_{p}$ in (8) can be replaced by one over $\gamma_{l}$. There is no Jacobian because the $\mathbb{Z}_{2}$ gauge orbits all have the same size. The constraints are now redundant, so $\gamma_{l}$ only appears in the action. This dependence can be removed by a change of variables $U_{l} \rightarrow \gamma_{l}^{-1} U_{l}$ which replaces $\alpha_{p}$ by the fixed $\zeta_{p}$. Due to the invariance of the Haar measure, the $\gamma_{l}$ sum can then be done trivially, and the Wilson partition function (6) is recovered.

\section{Twist in $\mathrm{SO}(3)$ LGT}

The bridge to $\mathrm{SO}(3)$ is now built by noting that, without the constraints, (8) becomes the Villain partition function of SO(3) LGT [24,

$$
Z_{\mathrm{SO}(3)}^{\mathrm{Villain}}=\sum_{\alpha_{p}= \pm 1} \int_{\mathrm{SU}(2)} \prod_{l} \mathrm{~d} U_{l} \exp \left(\frac{\beta}{2} \sum_{p} \alpha_{p} \operatorname{Tr}_{\mathrm{F}} U_{p}\right)=\int \prod_{l} \mathrm{~d} U_{l} \exp \left(-S_{\mathrm{V}}[U]\right)
$$

The Villain action, obtained after summation over $\alpha_{p}$,

$$
S_{\mathrm{V}}[U]=-\ln \left[2 \cosh \left(\frac{\beta}{2} \operatorname{Tr}_{\mathrm{F}} U_{p}\right)\right]
$$

is invariant under $U_{l} \rightarrow-U_{l}$ for each link separately, so (13) defines an SO(3) LGT, redundantly formulated in terms of $\mathrm{SU}(2)$ variables.

A comparison of Eqs. (8) and (13) exhibits the differences between $\mathrm{SU}(2)$ and $\mathrm{SO}(3)$ LGT. The first set of constraints in (8) shows that the $\mathrm{SO}(3)$ theory contains local degrees of freedom not present in $\mathrm{SU}(2)$ : the $\mathbb{Z}_{2}$ monopoles of the field $\alpha_{p}$. Loosely speaking, these monopoles are responsible for the bulk phase transition separating phases with and without monopole condensation in $\mathrm{SO}(3)$ LGT. Since $\alpha_{p}$ is only an auxiliary field, it is more appropriate to say that the $\mathrm{SO}(3)$ monopoles

$$
\eta_{c}=\prod_{p \in \partial c} \operatorname{sgn} \operatorname{Tr}_{\mathrm{F}} U_{p}
$$

condense at small $\beta$. In $\mathrm{SU}(2)$, these are strongly suppressed because negative plaquettes carry a large action.

The second set of constraints shows that, as opposed to $\mathrm{SU}(2)$, the partition function of $\mathrm{SO}$ (3) LGT with periodic boundary conditions contains all possible topological sectors, with arbitrary instanton number and twist $z$. This is as expected 
because in $\mathrm{SO}(3)$ all sectors are represented by transition functions with standard consistency conditions Eq. (41).

These considerations lead us to a natural definition of the twist $z$ in $\mathrm{SO}(3)$ on the lattice. In the formulation (8) of $\mathrm{SU}(2)$, the twist is given by Eq. (10) in terms of the auxiliary variables $\alpha_{p}$. In $\mathrm{SO}(3)$, it is better to define it in terms of $U_{p}$,

$$
\eta_{\mu \nu}=\frac{1}{L_{\rho} L_{\sigma}} \sum_{x_{\rho}, x_{\sigma}} \prod_{x_{\mu}, x_{\nu}} \operatorname{sgn} \operatorname{Tr}_{\mathrm{F}} U_{x, \mu \nu} \quad\left(\varepsilon_{\mu \nu \rho \sigma}=1\right) .
$$

The product measures the $\mathbb{Z}_{2}$ flux of the $\mathrm{SO}(3)$ gauge field $U / \mathbb{Z}_{2}$ through the $\mu \nu$ plane with fixed $x_{\rho}$ and $x_{\sigma}$. The apparent dependence on the sign of the SU(2) links $U_{l}$ drops out because each link appears in two plaquettes in the product. Thus $\eta_{\mu \nu}$ is a proper $\mathrm{SO}(3)$ observable. Contrary to (10), it can be defined in any $\mathrm{SO}(3)$ lattice gauge theory in formulations with or without auxiliary fields $\alpha_{p}$. The average over all parallel planes with a given orientation has been introduced because in $\mathrm{SO}(3)$, as opposed to $\mathrm{SU}(2)$, the flux can change from plane to plane due to the monopoles (15). This means that $\eta_{\mu \nu}$ can take fractional values $-1 \leq \eta_{\mu \nu} \leq 1$. A $\mathbb{Z}_{2}$-valued twist can be defined by dividing the range of $\eta_{\mu \nu}$ into two pieces:

$$
z_{\mu \nu}(\eta)=\operatorname{sgn}\left(\eta_{\mu \nu}-c_{\mu \nu}\right)
$$

with constant $c_{\mu \nu}$. Note that there is no symmetry $\eta_{\mu \nu} \leftrightarrow-\eta_{\mu \nu}$, so $c_{\mu \nu}=0$ is not singled out. The choice of $c_{\mu \nu}$ will be discussed in more detail later. The ambiguity in assigning a twist $z$ to a given configuration is similar to that of the usual topological charge in $\mathrm{SU}(2)$. It is caused by lattice artifacts (the monopoles $\eta_{c}$ ) which make the topological sectors connected. What is important is the behaviour in the continuum limit $\beta \rightarrow \infty$. Since monopoles are exponentially suppressed in this limit, one can hope that $z_{\mu \nu}(\eta)$ becomes independent of $c_{\mu \nu}$ there.

Consider first the naive (classical) continuum limit. In sectors with so-called orthogonal twist, $\kappa=0 \bmod N[\mathrm{cf}$. (5)] , the configuration with lowest action ("classical ground state") actually has zero action even though twist is present [25, 18. In $\mathrm{SU}(2)$, this "twist eater" can be constructed as

$$
U_{\mu}(x)= \begin{cases}\Gamma_{\mu} & \text { if } x_{\mu}=0 \\ \mathbb{1} & \text { otherwise }\end{cases}
$$

where the 4 matrices $\Gamma_{\mu}$ satisfy

$$
\Gamma_{\mu} \Gamma_{\nu} \Gamma_{\mu}^{\dagger} \Gamma_{\nu}^{\dagger}=z_{\mu \nu}^{*} \mathbb{1}
$$

This generates the plaquette field $U_{p}=\zeta_{p}^{*}$ which just cancels the factor $\zeta_{p}$ in the Wilson partition function with twist (6), so the action vanishes. The same configuration minimises the Villain action (14), because the latter is independent of the sign of $U_{p}$. The observable $\eta_{\mu \nu}$ (16), evaluated in this configuration, is identical to the $\mathrm{SU}(2)$ twist $z_{\mu \nu}$ of (19). The same holds true for small fluctuations around (18), 
so $\eta_{\mu \nu}$ has the proper naive continuum limit. For non-orthogonal twist, the lowestaction configurations are not known analytically, but it is expected that they still satisfy $\operatorname{sgn} \operatorname{Tr}_{\mathrm{F}} U_{p}=\alpha_{p}$ provided the lattice is fine enough. So $\eta_{\mu \nu}$ again coincides with the $\mathrm{SU}(2)$ twist.

Non-perturbatively, $\eta_{\mu \nu}$ is affected by lattice artifacts $\left(\eta_{c}\right)$ - just as the geometric definition of the instanton number is [26]. In our Monte-Carlo simulations, the distribution of $\eta_{\mu \nu}$ was always divided into two well-separated peaks close to \pm 1 (see Fig. 2 further below), so an unambiguous definition of twist was possible. For very large volumes, however, a (centre-blind) restriction of the plaquette angle might be necessary, like for geometric definitions of topological charges. The $\mathbb{Z}_{2}$-invariant choice

$$
\left|\operatorname{Tr}_{\mathrm{F}} U_{p}\right|>\varepsilon \text { where } \varepsilon>0
$$

makes the twist sectors disconnected (with $c_{\mu \nu}=0$ in (17)). ${ }^{4}$ For sufficiently large $\varepsilon$, lattice artifacts are completely suppressed and $\eta_{\mu \nu}= \pm 1$. In this case, however, the modified Villain theory becomes equivalent to an $\mathrm{SU}(2)$ theory with plaquette restriction $\operatorname{Tr}_{\mathrm{F}} U_{p}>\varepsilon$, summed over all twist sectors. The value of this theory for a comparison of $\mathrm{SO}(3)$ and $\mathrm{SU}(2)$ lattice gauge theory is thus questionable. We shall not apply a constraint like (201). For the parameters used in our simulations, the ambiguity in (17) has negligible impact.

\section{$5 \quad$ Negative Polyakov loops}

In this section, we would like to comment on the apparent weak coupling phase with negative adjoint Polyakov loop observed in Refs. [4, 5]. In these articles, $\mathrm{SO}(3)$ lattice gauge theory was studied with both the Wilson and the Villain action. For the lattice sizes used $\left(N_{t} \leq 8\right)$, only a single, first-order phase transition was found. This is the bulk phase transition below which monopoles condense. Although its position was found to be essentially independent of $N_{t}$, it was also interpreted as a deconfinement transition, because the expectation value of the adjoint Polyakov loop is very small below the transition and has a large modulus above. There, two different meta-stable "phases" were observed, depending on the initial configuration of the Monte-Carlo simulation. The two phases are distinguished by the sign of the adjoint Polyakov loop. The positive value can be understood as a standard deconfined phase where $\frac{1}{2} \operatorname{Tr}_{\mathrm{F}} P$ has an expectation value tending to \pm 1 in the limit $\beta \rightarrow \infty$. The adjoint loop $\operatorname{Tr}_{\mathrm{A}} P=4\left(\frac{1}{2} \operatorname{Tr}_{\mathrm{F}} P\right)^{2}-1$ therefore tends to +3 . No explanation was found for the other, "mysterious" phase. In [5], the correlator of two Polyakov loops and the response to an external field coupled to the Polyakov loop were found to be very similar in both phases, so the authors concluded that they were physically equivalent. At the critical coupling, tunnellings between both weak coupling phases and the unique strong coupling phase were observed.

\footnotetext{
${ }^{4}$ The discrete auxiliary variables $\alpha_{p}$ are of no concern here, as one can sum over them and work with the action (14).
} 


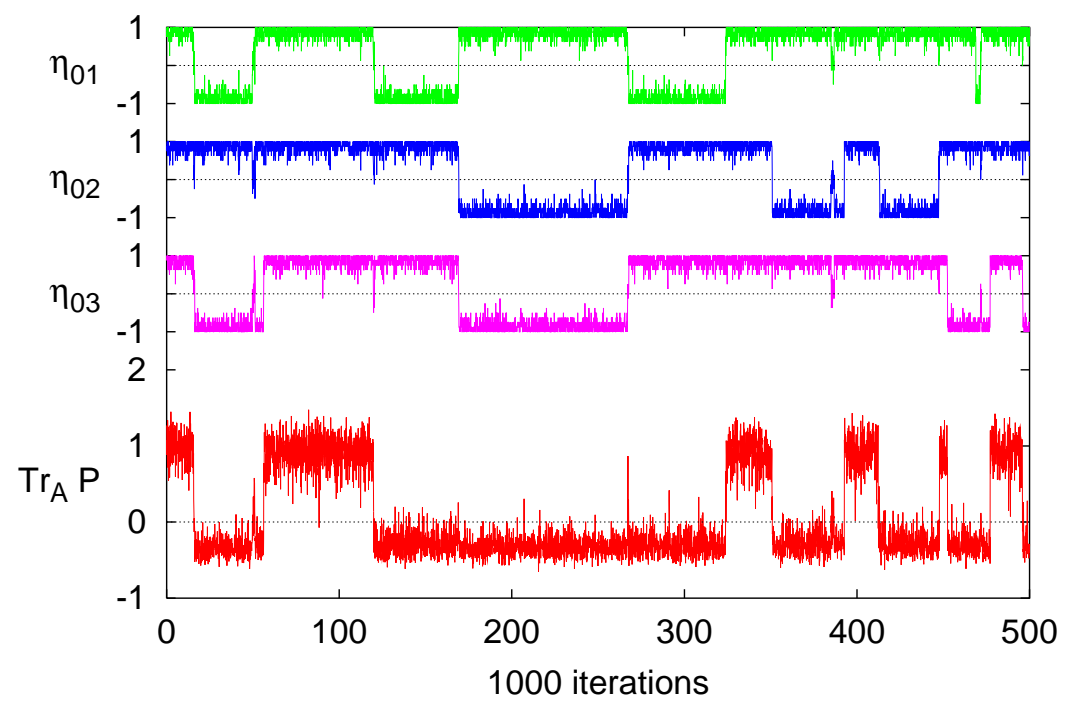

Figure 1: Monte Carlo history of the 3 electric twist variables (top) and the adjoint Polyakov loop (bottom) (4 ${ }^{4}$ lattice, $\beta=4.5$ ). Negative Polyakov loop values are always accompanied by one or more negative electric twists.

Based on the comparison between $\mathrm{SO}(3)$ and $\mathrm{SU}(2)$ LGT, we propose an explanation of the two weak coupling phases in terms of different twist. Consider the semi-classical limit. Without twist, all values of the Polyakov loop are compatible with zero classical action. These are the "toron" flat directions. The effective potential localises the fundamental Polyakov loop around two values which tend to \pm 1 in the continuum limit $\beta \rightarrow \infty$. Either way, this implies a positive adjoint Polyakov loop tending to +3 in the continuum limit. With non-vanishing twist, the flat directions disappear. For orthogonal twist, the configuration with smallest classical action is the twist eater (18). The (untraced) Polyakov loop of this configuration is just $\Gamma_{0}$. The simplest solution of (19) with a single electric twist $z_{03}=-1$ is $\Gamma_{0}=\mathrm{i} \sigma_{1}$ and $\Gamma_{3}=\mathrm{i} \sigma_{2}$, so in this case, the fundamental and adjoint Polyakov loops are $\operatorname{Tr}_{\mathrm{F}} \Gamma_{0}=0$ and $\operatorname{Tr}_{\mathrm{A}} \Gamma_{0}=-1$. In general, the twist eating matrices satisfy $(a)$ $\operatorname{Tr}_{\mathrm{A}} \Gamma_{0}=-1$ if $z_{0 i}=-1$ for some $i$, or $(b) \operatorname{Tr}_{\mathrm{A}} \Gamma_{0}=+3$ if $z_{0 i}=0$ for all $i$, but $z_{i j} \neq 0$ for some $i, j$. The arguments leading to this known result are collected in the Appendix. This classical consideration suggests that the phase with negative Polyakov loop is one with electric twist. This can be verified numerically. Figure 1 shows the Monte-Carlo history of the 3 electric twist variables $\eta_{0 i}$ as well as the adjoint Polyakov loop on a $4^{4}$ lattice at $\beta=4.5$, just above the bulk phase transition at $\beta \approx 4.45$. The system tunnels between phases with positive and negative Polyakov loop. The negative phases always have one or more electric twists and the positive phases none. The twist observable (16) has now been studied also with the $\mathrm{SO}(3)$ Wilson action, and meta-stable phases with different $z_{0 i}$ have similarly been found [27].

We would like to remark that semi-classically, the above argument is not conclusive. The twist eater has a Polyakov loop $\operatorname{Tr}_{\mathrm{F}} P=0$, i.e. on the maximum of the 
one-loop effective potential. Therefore, it might not be the dominant semi-classical configuration; a configuration with a $\mathbb{Z}_{N}$ interface (Euclidean "domain wall"), interpolating between the minima of the effective potential, might have a lower effective action. Outside of the interface, the adjoint Polyakov loop is positive. For large volumes, its spatial average therefore is positive, too. If the configuration with an interface indeed has lower effective action, the average Polyakov loop will be positive even in the presence of electric twist, provided the spatial extent of the lattice is sufficiently large compared to the interface thickness.

\section{$6 \quad$ Twist free energies}

We shall now measure the free energy of various twists, both in $\mathrm{SO}(3)$ and in $\mathrm{SU}(2)$. The results are used to calibrate the $\mathrm{SO}(3)$ lattice spacing. We choose the $\mathrm{SO}(3)$ coupling $\beta=4.5$ just above the bulk phase transition at $\beta \approx 4.45$ in order to find the largest lattice spacing available in the weak coupling phase of $\mathrm{SO}(3)$.

The twist free energies $F(z)$ are defined in terms of partition functions with and without twist,

$$
F(z)=-\ln \frac{Z(z)}{Z(1)}
$$

For electric twist $\left(z_{0 i} \neq 1\right), F(z)$ is an order parameter for the confinement-deconfinement transition [12, 13]: for large volumes, it tends to 0 in the confined phase, while diverging as $F \sim \tilde{\sigma} L^{2}$ with the spatial extent $L$ in the deconfined phase. $\tilde{\sigma}$ is the dual string tension and depends on the temperature. In $\mathrm{SU}(2)$, the partition functions $Z(z)$ and $Z(1)$ are defined by imposing twisted and periodic boundary conditions, respectively. In $\mathrm{SO}(3)$, all twists are summed over within periodic boundary conditions, and twist is an observable like the usual topological charge in $\mathrm{SU}(2)$. The partition functions are defined using (17),

$$
Z(z)=Z_{\mathrm{tot}}\left\langle\prod_{\mu<\nu} \delta\left(z_{\mu \nu}(\eta), z_{\mu \nu}\right)\right\rangle
$$

where $Z_{\text {tot }}=\sum_{\left\{z_{\mu \nu}\right\}} Z(z)$. In order to determine the value $c_{\mu \nu}$ of Eq. (17) which divides trivial and non-trivial twist, we use the "density of states" as a function of a single $\eta_{\mu \nu}$

$$
P_{\mu \nu}(x)=\left\langle\delta\left(\eta_{\mu \nu}-x\right)\right\rangle
$$

Figure 2 shows $P_{0 i}$ for a $8^{3} \times 4$ lattice at $\beta=4.5$. We adjust $c_{\mu \nu}$ to the minimum of $P_{\mu \nu}$, which is suppressed by several orders of magnitude with respect to both maxima, so the precise choice of $c$ has negligible impact, and twist can indeed be defined unambiguously, as claimed above. This has been confirmed numerically by comparing the above choice of $c$ with $c=0$. The results agree within statistical errors.

The strong suppression of intermediate values of $\eta_{0 i}$ turns out to be a practical problem: a simple Metropolis algorithm is not capable of changing the electric twist 


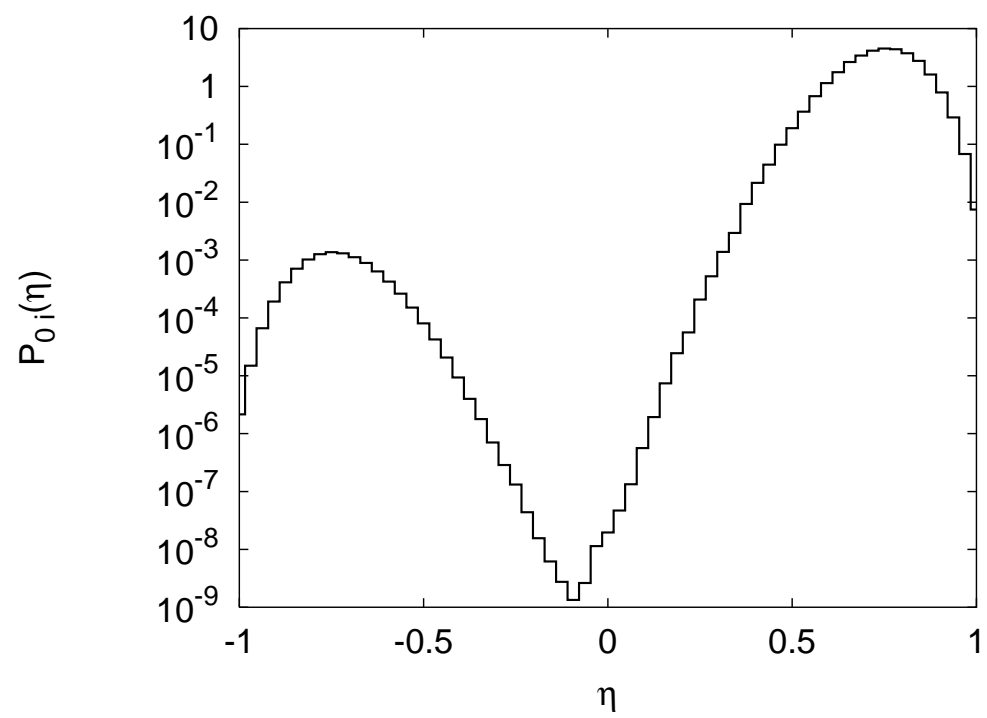

Figure 2: "Density of states" $P_{0 i}$ of an electric twist $\left(8^{3} \times 4\right.$ lattice, $\left.\beta=4.5\right)$.

with sufficient probability on lattices larger than $4^{4}$ sites. To achieve ergodicity across twist sectors, we use a variant of the multicanonical algorithm [28, applied to $\eta_{0 i}$ instead of the action (a "multitwist" algorithm so to speak). The depletion in the density of states as a function of $\eta_{0 i}$ is removed by adding a bias $\Delta S\left(\eta_{01}, \eta_{02}, \eta_{03}\right)$ to the action, so configurations are generated with probability $\frac{1}{Z} \mathrm{e}^{-S-\Delta S}$. The MonteCarlo process then amounts to a free diffusion across twist sectors, with dynamics accelerated exponentially. The modification in probability is corrected by an inverse factor $\mathrm{e}^{\Delta S}$ in the observables.

The bias $\Delta S\left(\eta_{01}, \eta_{02}, \eta_{03}\right)$ can be represented as a 3 -dimensional table since $\eta_{0 i}$ only takes $L^{2}+1$ discrete values (cf. Eq. (16) ). This table is constructed iteratively, using converged values on small lattices to form starting values on larger ones. Since the barrier is expected to grow approximately quadratically in the system size, the initial bias is scaled as $\Delta S_{L^{\prime}}=\left(L^{\prime} / L\right)^{2} \Delta S_{L}$. Such a table is displayed in Fig. 3, for an $8^{3} \times 4$ lattice at $\beta=4.5$. If $\Delta S$ were a sum of 3 functions of only one variable, the iterative determination would be greatly simplified. Alas, Fig. 3 shows that this is not the case. Note that, although $\beta$ has been chosen as small as possible (the bulk transition to the strongly coupled phase occurs at $\beta \approx 4.45$ ), the necessary enhancement of the saddle points reaches $10^{12}$, as seen more clearly in Fig. 4, which is a 1-dimensional cut of the same table along its diagonal (from $\eta_{0 i}=(-1,-1,-1$ ) to $(1,1,1))$. There, the resulting flatness of the Monte Carlo sampling probability is clearly visible. The measured density of states shows two well-separated peaks, corresponding to twist sectors $(1,1,1)$ (analogous to $\left.S U(2)_{\mathrm{pbc}}\right)$ and $(-1,-1,-1)$ (analogous to $S U(2)_{\mathrm{tbc}}$ in all 3 temporal planes). The strong suppression of the saddle point confirms that ordinary Monte Carlo sampling would remain hopelessly "stuck" in one sector, leading to the "phases" observed in earlier studies. However, in spite of the great multicanonical acceleration, the Monte Carlo evolution of the 


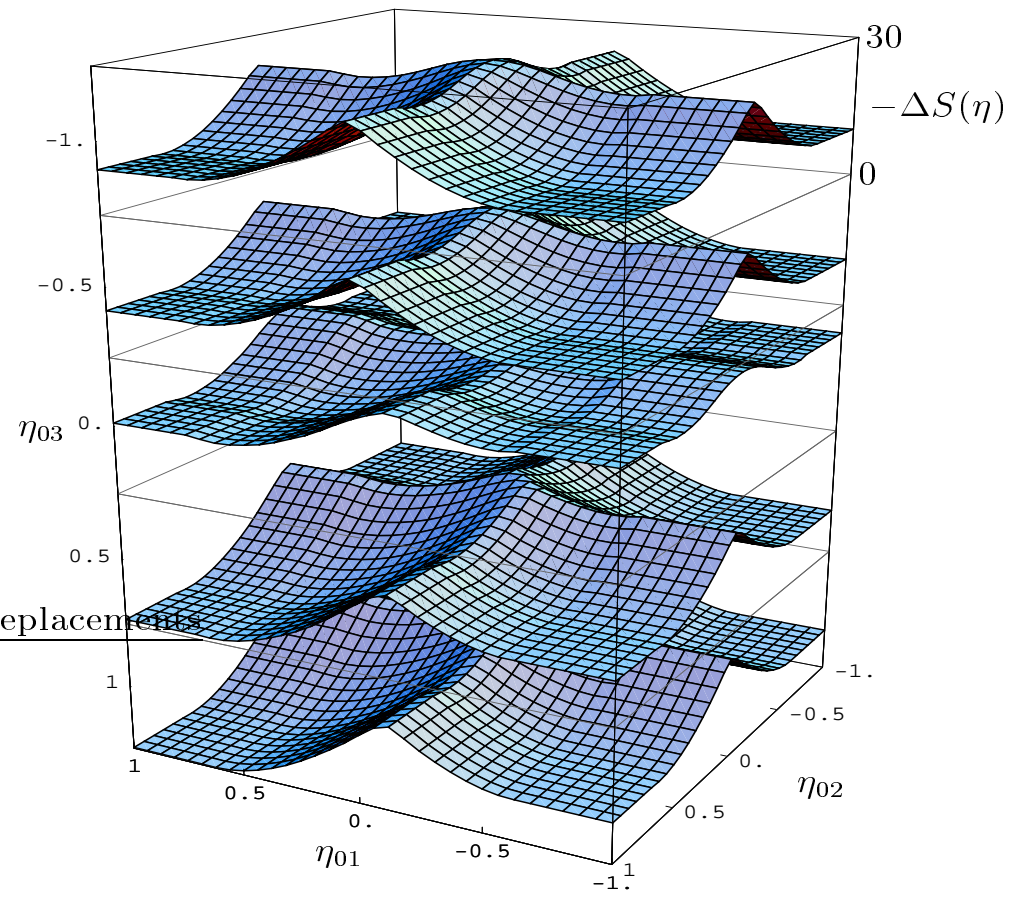

Figure 3: The three-dimensional reweighting table used in our multicanonical Monte Carlo. Five sections $-\Delta S\left(\eta_{01}, \eta_{02}\right)$ are shown for fixed $\eta_{03}$. No twist is at bottom left, twist in all 3 planes at top right. The table enhances the probability of sampling the saddles between twist sectors. Its entries $\mathrm{e}^{-\Delta S}$ vary by 12 orders of magnitude $\left(8^{3} \times 4\right.$ lattice, $\left.\beta=4.5\right)$.

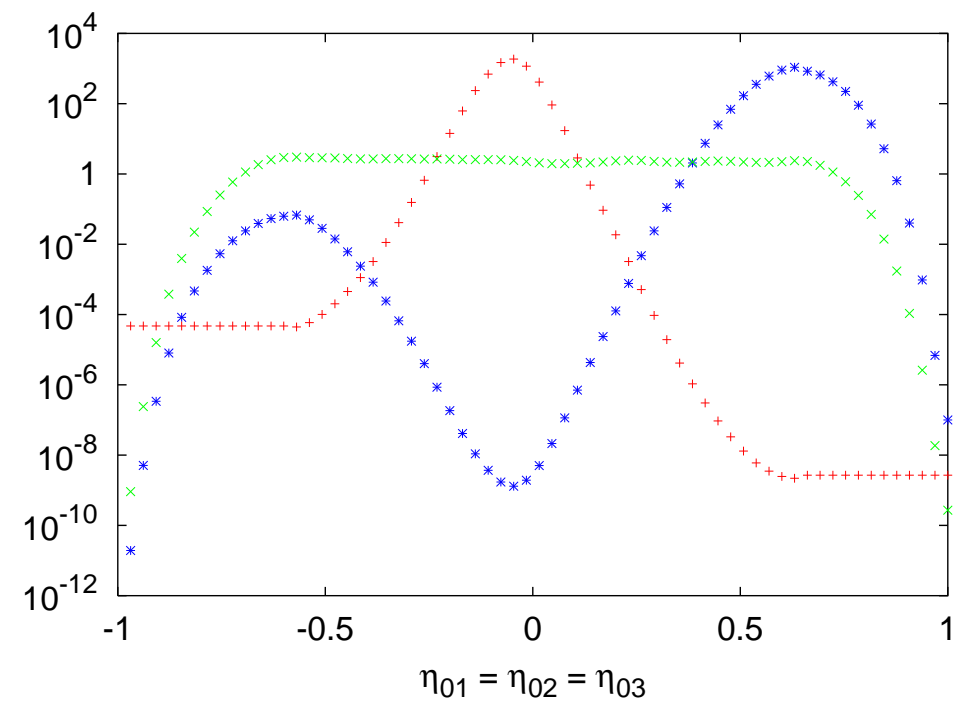

Figure 4: Cut of the three-dimensional reweighting table along its diagonal $($ red +$)$. The result of multicanonical Monte Carlo sampling is a nearly flat histogram (green $\mathrm{x})$. The density of states shows the dominant twist-0 and the smaller twist-3 sectors (blue $\left.{ }^{*}\right)$. 


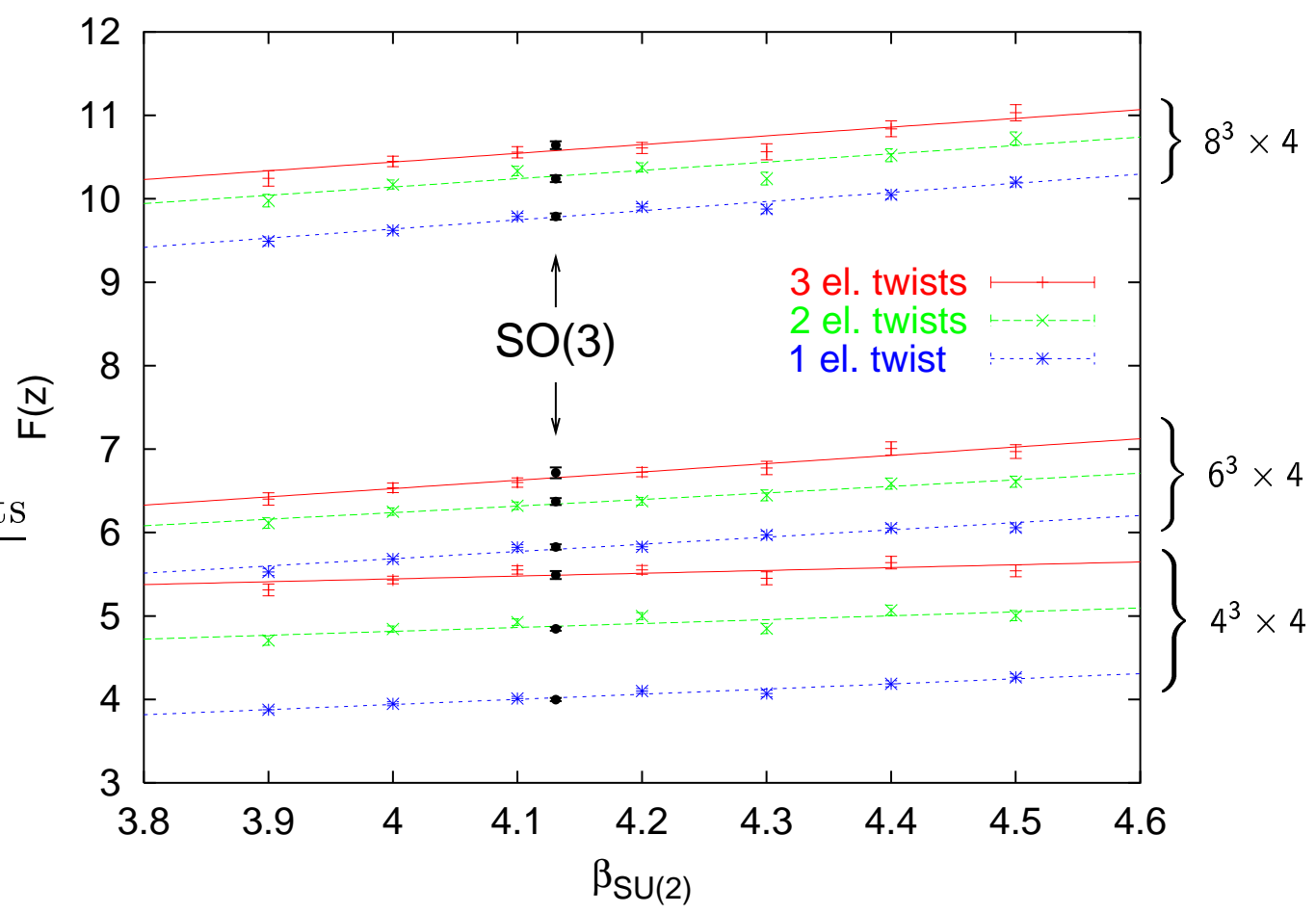

Figure 5: Comparison of the twist free energies $F(z)$ between $S O(3)$ at $\beta=4.5$ and $S U(2)$ at various $\beta$. Electric twist in 1,2 and 3 planes is considered, on lattices of size $4^{3} \times 4,6^{3} \times 4$ and $8^{3} \times 4$. One finds $\beta_{S O(3)}=4.5 \longleftrightarrow \beta_{S U(2)}=4.13(3)$.

twist variables $\eta_{0 i}$ is still slow, and simulating a $10^{3} \times 4$ lattice remains beyond the edge of our computer resources.

This prevents us from reaching spatial sizes large enough for a reliable determination of the dual string tension [8]. Nevertheless, we can still compare the free energies for various small volumes with those obtained in $\mathrm{SU}(2)$ with the method of 9. This also allows us to calibrate the $S O(3)$ lattice spacing.

The twisted free energies $F(z)$ are continuum quantities, which depend upon the spatial size $L$ and the temperature $T$. If the $S O(3)$ and $S U(2)$ theories represent the same continuum physics, then to each $\beta_{S O(3)}$ should correspond a value $\beta_{S U(2)}$, which yields the same lattice spacing and thereby the same $F(z)$ for equal lattice sizes, modulo small lattice artifacts. We test this statement in Fig. 5 , The 9 free energies $F(z)$ for 1,2 or 3 electric twists measured on $4^{3} \times 4,6^{3} \times 4$ and $8^{3} \times 4$ lattices in $S O(3)$ at $\beta_{S O(3)}=4.5$ are compared with similar quantities measured in $S U(2)$ at different values of $\beta_{S U(2)}$. The straight lines show a linear interpolation in $\beta_{S U(2)}$ of the $S U(2)$ data. One observes a very good match $\left(\chi^{2} /\right.$ dof $\left.\approx 1.35\right)$ of all 9 observables, for $\beta_{S U(2)} \approx 4.13(3)$.

Thus, $a^{-1}\left(\beta_{S O(3)}=4.5\right) \approx a^{-1}\left(\beta_{S U(2)}=4.13\right)$, which is about $200 \mathrm{GeV}$ ! As conventional wisdom asserts, our $S O(3)$ lattice is very fine indeed. It cannot be made coarser because of the bulk transition at $\beta \approx 4.45$. Therefore, to reach low 
temperatures $T<T_{c}$ and probe the confined phase would require a lattice of size $\mathcal{O}\left(700^{4}\right)$, far beyond what is currently achievable. We would like to emphasise that the scale of $200 \mathrm{GeV}$ is in no way related to continuum physics. It is merely due to the bulk phase transition of the $\mathrm{SO}(3)$ lattice gauge theory beyond which lattice artifacts dominate, and gives a lower bound on the cutoffs one can use. This value can be shifted by suppressing (or enhancing) lattice artifacts [27].

Note the closeness of the matched $S O(3)-S U(2)$ bare couplings $\left(\frac{4}{g^{2}}=4.5\right.$ vs. 4.13). This should come as no surprise. Lattice perturbation theory is identical between the $S U(2)$ Wilson action and the $S O(3)$ Villain action: the difference resides in the $\alpha$-monopoles, which do not appear in the perturbative expansion. Therefore, $\Lambda_{\text {lattice }}$ is the same in both theories, and one should expect similar values for the nonperturbatively matched $\beta$ 's. The $\alpha$-monopoles disorder the $S O(3)$ theory slightly, which is why the $S U(2)$ matching $\beta$ is slightly smaller.

\section{Discussion and conclusions}

We have seen that a Monte-Carlo simulation of the confined phase of $\mathrm{SO}(3)$ lattice gauge theory with the Villain action is not feasible in the near future because a bulk phase transition imposes an upper bound on the lattice spacing. This is expected to be true of the Wilson action as well. Since the bulk phase transition is caused by $\mathbb{Z}_{2}$ monopoles, the question arises whether the confined phase can be reached with a modified $\mathrm{SO}(3)$ action suppressing them. A chemical potential can move the transition to stronger coupling and larger lattice spacing. There are three kinds of monopoles in the Villain theory, $\alpha_{c}, \eta_{c}[$ cf. Eqs. (9) and (15)] $]$ and $\sigma_{c} \equiv \alpha_{c} \eta_{c}$, and one can introduce chemical potentials for all: ${ }^{5}$

$$
\begin{gathered}
Z\left(\beta, \lambda_{\alpha}, \lambda_{\eta}, \lambda_{\sigma}\right)=\sum_{\alpha_{p}= \pm 1} \int \prod_{l} \mathrm{~d} U_{l} \exp \left[-S\left(\beta, \lambda_{\alpha}, \lambda_{\eta}, \lambda_{\sigma}\right)\right] \\
S\left(\beta, \lambda_{\alpha}, \lambda_{\eta}, \lambda_{\sigma}\right)=-\frac{\beta}{2} \sum_{p} \alpha_{p} \operatorname{Tr}_{\mathrm{F}} U_{p}-\lambda_{\alpha} \sum_{c} \alpha_{c}-\lambda_{\eta} \sum_{c} \eta_{c}-\lambda_{\sigma} \sum_{c} \alpha_{c} \eta_{c} .
\end{gathered}
$$

The parameter $\lambda_{\alpha}$ (for $\lambda_{\eta}=\lambda_{\sigma}=0$ ) interpolates between Villain $\mathrm{SO}(3)$ and Wilson $\mathrm{SU}(2)$ [cf. (8)]. It has been studied by Halliday and Schwimmer [30] who found that the bulk transition is indeed moved to smaller $\beta$ and disappears $(\beta=0)$ around $\lambda_{\alpha}^{\mathrm{c}} \approx 0.95$, see Fig. 6. The deconfinement transition can therefore be studied on small lattices for $\lambda_{\alpha}>\lambda_{\alpha}^{c}$ [31. However, since the limit $\lambda_{\alpha} \rightarrow \infty$ reduces to the $\mathrm{SU}(2)$ theory, it is clear that $\lambda_{\alpha} \neq 0$ reintroduces the $\mathbb{Z}_{2}$ part of $\mathrm{SU}(2)$ missing in $\mathrm{SO}(3)$. For $\lambda_{\alpha} \neq 0$, (25) is not really an $\mathrm{SO}(3)$ action, but an $\mathrm{SU}(2)$ action in disguise. It is not suited for verifying the equivalence of $\mathrm{SO}(3)$ and $\mathrm{SU}(2)$. This is expected to be true also of the theory with $\lambda_{\sigma} \neq 0$, since this also affects the variables $\alpha_{p}$ such that they cannot be summed over trivially.

\footnotetext{
${ }^{5}$ The effect of $\lambda_{\alpha}$ and $\lambda_{\sigma}$ (with $\lambda_{\eta}=0$ ) has been studied in [29].
} 


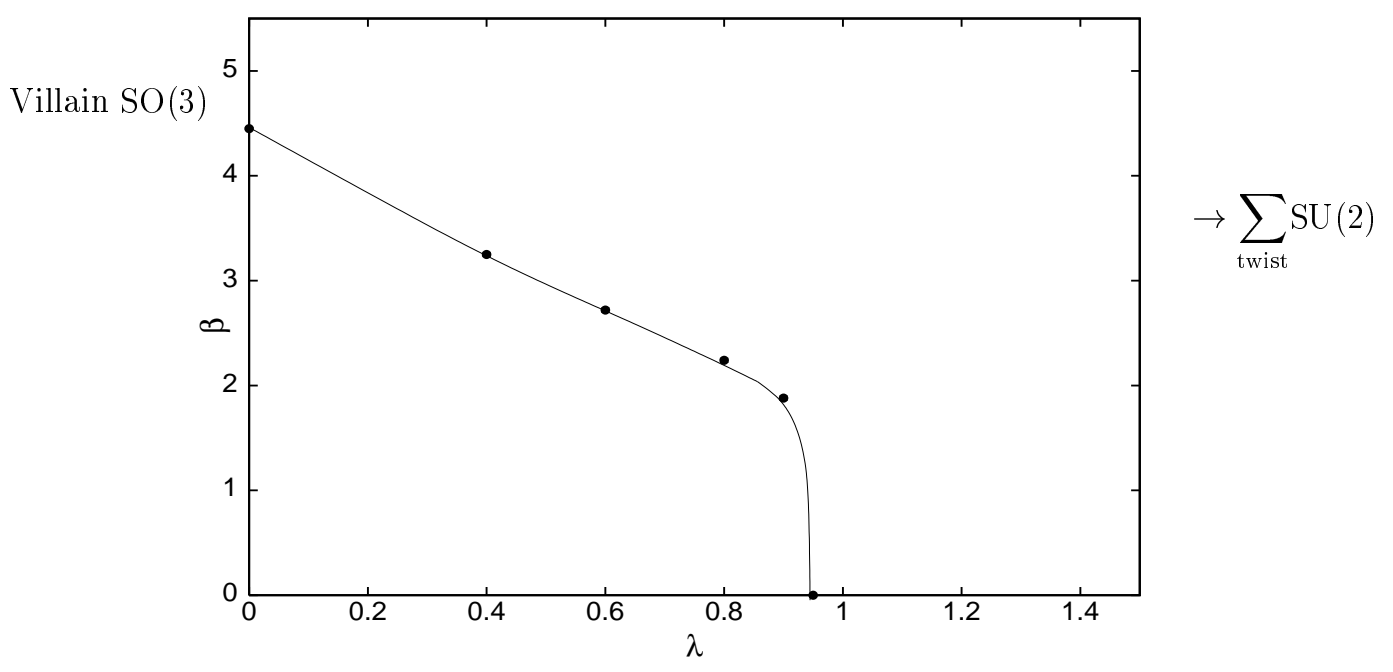

Figure 6: Phase diagram of Villain $\mathrm{SO}(3)$ with $\alpha$-monopole suppression, from [31].

Note that $\lambda_{\alpha}$ provides an analytic path between the weak coupling phase of Villain $\mathrm{SO}(3)$ and $\mathrm{SU}(2)$, since no phase transition except the above-mentioned monopole condensation transition has been found [30, 31]. This is a strong argument in favour of the equivalence of the two theories in the continuum limit. It is likely that an analytic path can be found in the same way for other $\mathrm{SO}(3)$ actions (e.g. the Wilson action): any $\mathrm{SO}(3)$ theory can be formulated with auxiliary plaquette variables à la Villain, and related to an $\mathrm{SU}(2)$ theory by a chemical potential for $\alpha$-monopoles. The action of this $\mathrm{SU}(2)$ theory is to some extent arbitrary: only the centre-symmetric part of the plaquette weight $\exp \left[-S_{p}\left(U_{p}\right)\right]$ is required to be equal to that of the $\mathrm{SO}(3)$ theory. The anti-symmetric part is only restricted by the positivity of the weight. We expect the monopole condensation transition to move to small $\beta$ with a suitable choice of antisymmetric part for most theories, so that an analytic path will exist.

The remaining option, $\lambda_{\eta} \neq 0$ (but $\lambda_{\alpha}=\lambda_{\sigma}=0$ ) does not reintroduce $\mathrm{SU}(2)$ degrees of freedom: $\alpha_{p}$ can still be summed over, and one obtains a manifestly centre-invariant formulation in terms of $\mathrm{SO}(3)$ matrices. $\lambda_{\eta}$ can also be introduced in the Wilson $\mathrm{SO}(3)$ action. There, like $\lambda_{\alpha}$ in the Villain theory, it shifts the bulk transition to smaller, eventually negative $\beta$ 32. One expects $\lambda_{\eta}$ to have the same effect in the Villain theory also. However, for $\lambda_{\eta} \rightarrow \infty$ the theory now becomes equivalent to an $\mathrm{SU}(2)$ positive plaquette model: if $\eta_{c} \equiv 1$, the plaquette field $\eta_{p}$ can be expressed in terms of a link field, $\eta_{p}=\prod_{\partial p} \gamma_{l}{ }^{6}$ A change of integration variables $U_{l} \rightarrow \gamma_{l} U_{l}$ makes the plaquette variables trivial, $\eta_{p}=\operatorname{sgn} \operatorname{Tr} U_{p}=+1$. This is just the positive plaquette constraint $\operatorname{Tr} U_{p} \geq 0$. After summing over $\alpha_{p}$ (still $\left.\lambda_{\alpha}=0\right)$, we obtain a positive plaquette model with action $S=-\sum_{p}\left\{\frac{\beta}{2} \operatorname{Tr} U_{p}+\ln [1+\right.$ $\left.\left.\exp \left(-\beta \operatorname{Tr} U_{p}\right)\right]\right\}$. This modification of action is not expected to have a significant

\footnotetext{
${ }^{6}$ In the presence of twist, a representative has to be introduced for each twist, like in Eq. (12).
} 
effect on the deconfinement transition. Therefore, comparing $\mathrm{SU}(2)$ with Wilson action and $\mathrm{SO}(3)$ with action $S\left(\beta, 0, \lambda_{\eta}, 0\right), \lambda_{\eta}>0$ is little more than comparing the former with a positive plaquette version of itself. For the latter, equivalence with Wilson SU(2) seems established [33. Moreover, maintaining ergodicity is even more difficult than without monopole suppression because the barriers between different twist sectors are even higher.

We conclude that the equivalence of $\mathrm{SO}(3)$ and $\mathrm{SU}(2)$ lattice gauge theory cannot be directly verified by simulation at the present time. The arguments presented in the literature against such an equivalence, however, can be invalidated: an analytic path between the theories at weak coupling exists under weak assumptions and the phase with negative adjoint Polyakov loop observed in $\mathrm{SO}(3)$ turns out to correspond to sectors with electric twist in $\mathrm{SU}(2)$. The only difference between the two theories is that a definite twist sector is selected via the choice of boundary conditions in $S U(2)$, whereas all sectors are automatically summed over within periodic boundary conditions in $S O(3)$. Also the absence of the centre symmetry in $\mathrm{SO}(3)$ is not a problem: it is not needed to characterise the deconfinement transition. The (spatial) centre vortex - or electric twist - free energy can be used as an order parameter in both theories. At the very high temperatures accessible to simulations to date, the centre vortex free energies of $\mathrm{SO}(3)$ and $\mathrm{SU}(2)$ are numerically compatible, provided the scales are adjusted between both theories.

Finally, one can try to generalise the lesson learnt here about the structure of the gauge group $G$ necessary for confinement. $S O(3)$ shows that a non-trivial centre is not required (cf. [6]). On the other hand, the existence of the dual string tension characterising the deconfined phase arises from that of 't Hooft loops or twist sectors. Those in turn follow from the non-trivial first homotopy group $\pi_{1}[G / \operatorname{Centre}(G)]$, which is the same for $S U(2)$ and $S O(3)$. A non-trivial $\pi_{1}[G / \operatorname{Centre}(G)]$, or more precisely $\pi_{1}\left[G / Z_{G}\right]$, where $Z_{G}$ is the discrete part of the centre of $G$, is necessary for a dual string tension to be defined. The non-trivial elements of this group are the centre vortex (or twist) excitations. There are only 3 (simple) non-Abelian Lie groups for which $\pi_{1}\left[G / Z_{G}\right]$ is trivial: $G_{2}, F_{4}$ and $E_{8}$. The absence of a dual string tension in the corresponding gauge theories prompts speculations about the nature of the confinement/deconfinement phase transition. The study of $\mathrm{G}_{2}$ proposed in 34 will be interesting in this connection.

\section{Acknowledgements}

We are grateful to A. Barresi, G. Burgio, M. Müller-Preussker and L. von Smekal for discussions. 


\section{Appendix: Twist and Polyakov loops}

Here, we shall review the relation between twist and Polyakov loops for general twist eaters, working out the arguments sketched in [35].

Consider a twist eater (18) given in terms of 4 constant matrices $\Gamma_{\mu} \in \mathrm{SU}(2)$ which satisfy the relations (19),

$$
\Gamma_{\mu} \Gamma_{\nu}=\mathrm{e}^{\mathrm{i} \pi n_{\mu \nu}} \Gamma_{\nu} \Gamma_{\mu}
$$

with $n_{\mu \nu}=0,1$. These relations define a group, and the matrices $\Gamma_{\mu}$ can be considered a (non-unique) 2-dimensional representation of this group. The simple argument below depends on the fact that, for $\mathrm{SU}(2)$, all such representations are irreducible unless $n_{\mu \nu}=0$ for all $\mu, \nu$.

A generalised Polyakov loop in the fundamental representation which winds around several directions, successively $s_{\mu}$ times around the $\mu$-direction, can be defined as

$$
\begin{aligned}
P_{\mathrm{F}}(s) & =\frac{1}{2} \operatorname{Tr}_{\mathrm{F}} \Gamma(s) \\
\Gamma(s) & =\Gamma_{3}^{s_{3}} \Gamma_{2}^{s_{2}} \Gamma_{1}^{s_{1}} \Gamma_{0}^{s_{0}} .
\end{aligned}
$$

It is not necessary to consider other orders of the factors $\Gamma_{\mu}$, since, by Eq. (26)), the order only affects the sign of $P_{\mathrm{F}}(s)$. Furthermore, irreducibility implies $\Gamma_{\mu}^{2}= \pm 1$ [35], so $\left|P_{\mathrm{F}}(s)\right|$ depends on $s_{\mu}$ only modulo 2 and it is sufficient to consider $s_{\mu} \in\{0,1\}$.

We show that, provided $n \neq 0,\left|P_{\mathrm{F}}(s)\right|$, and thus $P_{\mathrm{A}}(s)$, only depend on $n_{\mu \nu}$ and not on the particular representation $\Gamma_{\mu}$. More precisely,

$$
\left|P_{\mathrm{F}}(s)\right|=\left\{\begin{array}{lll}
0 & \text { if } \sum_{\nu} n_{\mu \nu} s_{\nu} \neq 0 & (\bmod 2) \\
1 & \text { if } \sum_{\nu} n_{\mu \nu} s_{\nu}=0 & (\bmod 2)
\end{array}\right.
$$

To see this, note that Eq. (26) implies

$$
\Gamma\left(s^{\prime}\right) \Gamma(s)=\mathrm{e}^{\mathrm{i} \pi s^{\prime} \cdot n s} \Gamma(s) \Gamma\left(s^{\prime}\right)
$$

where $s^{\prime} \cdot n s=\sum_{\mu \nu} s_{\mu}^{\prime} n_{\mu \nu} s_{\nu}$. Now, $n s \neq 0(\bmod 2)$ implies that $s^{\prime} \cdot n s=1(\bmod 2)$ for some $s^{\prime}$ and thus

$$
\Gamma\left(s^{\prime}\right) \Gamma(s)=-\Gamma(s) \Gamma\left(s^{\prime}\right) .
$$

There is a theorem that says that the relation (31) for two invertible matrices $\Gamma(s)$ and $\Gamma\left(s^{\prime}\right)$ implies $\operatorname{Tr}_{\mathrm{F}} \Gamma(s)=0$ (Lemma 1 of 35, Sec. 4.3). This proves the first part of (29). If $n s=0(\bmod 2)$, on the other hand, $\Gamma(s)$ commutes with all $\Gamma\left(s^{\prime}\right)$, in particular the generators $\Gamma_{\mu}$. If $n \neq 0$, irreducibility implies that $\Gamma(s)= \pm 1$, proving the second part.

It is straightforward to convince oneself that the values of $\left|P_{\mathrm{F}}(s)\right|$ for $s_{\mu}=0,1$ determine the twist $n_{\mu \nu}$ uniquely through Eq. (29), see Table 1.

For vanishing twist, all values of the Polyakov loop have minimal classical action, since (26) is satisfied by arbitrary diagonal matrices $\Gamma_{\mu}$. These are the so-called "toron" modes [36]. 


\begin{tabular}{ccccccccc}
$|\vec{m}|^{2}$ & $|\vec{k}|^{2}$ & $P_{0}$ & $|\vec{P}|^{2}$ & $\vec{P} \cdot \vec{M}$ & $|\vec{M}|^{2}$ & $\vec{M} \cdot \vec{K}$ & $|\vec{K}|^{2}$ & $\vec{P} \cdot \vec{K}$ \\
\hline 0 & 1 & 0 & 2 & 0 & 1 & 0 & 0 & 0 \\
0 & 2 & 0 & 1 & 1 & 1 & 0 & 0 & 0 \\
0 & 3 & 0 & 0 & 0 & 3 & 0 & 0 & 0 \\
\hline 1 & 0 & 1 & 1 & 0 & 0 & 0 & 1 & 1 \\
1 & 1 & 0 & 1 & 0 & 0 & 0 & 1 & 0 \\
1 & 2 & 0 & 1 & 0 & 0 & 0 & 0 & 0 \\
\hline 2 & 0 & 1 & 0 & 0 & 1 & 0 & 0 & 0 \\
2 & 1 & 0 & 0 & 0 & 1 & 0 & 2 & 0 \\
2 & 2 & 0 & 0 & 0 & 1 & 1 & 1 & 0 \\
2 & 3 & 0 & 0 & 0 & 1 & 0 & 0 & 0 \\
\hline 3 & 0 & 1 & 0 & 0 & 0 & 0 & 0 & 0 \\
3 & 2 & 0 & 0 & 0 & 0 & 0 & 1 & 0
\end{tabular}

Table 1: Multiple Polyakov loops for twist eaters with twist $n_{i j}=\varepsilon_{i j k} m_{k}, n_{0 i}=$ $k_{i}$. Polyakov loops winding around up to 2 cycles of the torus are considered: $P_{\mu}=\left|P_{\mathrm{F}}\left(e_{\mu}\right)\right|, K_{i}=\left|P_{\mathrm{F}}\left(e_{0}+e_{i}\right)\right|$ and $M_{k}=\left|P_{\mathrm{F}}\left(e_{i}+e_{j}\right)\right|$ with $\{i, j, k\}=\{1,2,3\}$. This information is sufficient to determine the twist up to a permutation of space coordinates. The individual components can be obtained from the components of $\vec{P}, \vec{K}, \vec{M}$ and $P_{0}$, if desired. Some twists are missing because twist eaters only exist for orthogonal twist.

\section{References}

[1] K. Langfeld and H. Reinhardt, The Stefan-Boltzmann law: SU(2) versus SO(3) lattice gauge theory, hep-lat/0001009.

[2] G. Bhanot and M. Creutz, Variant actions and phase structure in lattice gauge theory, Phys. Rev. D24 (1981) 3212.

[3] L. Caneschi, I. G. Halliday and A. Schwimmer, The phase structure of mixed lattice gauge theories, Nucl. Phys. B200 (1982) 409.

[4] S. Cheluvaraja and H. S. Sharathchandra, Finite temperature properties of mixed action lattice gauge theory, hep-lat/9611001.

[5] S. Datta and R. V. Gavai, Phase transitions in SO(3) lattice gauge theory, Phys. Rev. D57 (1998) 6618-6624, hep-lat/9708026.

[6] B. Svetitsky and L. G. Yaffe, Critical behavior at finite temperature confinement transitions, Nucl. Phys. B210 (1982) 423.

[7] A. V. Smilga, Are Z(N) bubbles really there?, Ann. Phys. 234 (1994) 1-59.

[8] G. 't Hooft, On the phase transition towards permanent quark confinement, Nucl. Phys. B138 (1978) 1. 
[9] Ph. de Forcrand, M. D'Elia and M. Pepe, A study of the 't Hooft loop in SU(2) Yang-Mills theory, Phys. Rev. Lett. 86 (2001) 1438, hep-lat/0007034.

[10] G. 't Hooft, A property of electric and magnetic flux in nonabelian gauge theories, Nucl. Phys. B153 (1979) 141.

[11] T. G. Kovács and E. T. Tomboulis, Computation of the vortex free energy in SU(2) gauge theory, Phys. Rev. Lett. 85 (2000) 704-707, hep-lat/0002004.

[12] Ph. de Forcrand and L. von Smekal, 't Hooft loops, electric flux sectors and confinement in SU(2) Yang-Mills theory, Phys. Rev. D66 (2002) 011504, hep-lat/0107018.

[13] L. von Smekal and Ph. de Forcrand, Electric and magnetic fluxes in SU(2) Yang-Mills theory, hep-lat/0209149.

[14] $\mathrm{Ph}$. de Forcrand and O. Jahn, $S O(3)$ versus $S U(2)$ lattice gauge theory, NATO Sci. Ser. II/83 (2002) 287, hep-lat/0205026.

[15] Ph. de Forcrand and O. Jahn, Vortex free energies in $S O(3)$ and $S U(2)$ lattice gauge theory, hep-lat/0209060.

[16] S. Sedlacek, A direct method for minimizing the Yang-Mills functional over four manifolds, Commun. Math. Phys. 86 (1982) 515-527.

[17] C. Nash, Gauge potentials and bundles over the four torus, Commun. Math. Phys. 88 (1983) 319.

[18] J. Groeneveld, J. Jurkiewicz and C. P. Korthals Altes, Twist as a probe for phase structure, Phys. Scripta 23 (1981) 1022.

[19] D. R. Lebedev, M. I. Polikarpov and A. A. Roslyi, Gauge fields on the continuum and lattice tori, Nucl. Phys. B325 (1989) 138.

[20] G. Mack and V. B. Petkova, Z2 monopoles in the standard SU(2) lattice gauge theory model, Zeit. Phys. C12 (1982) 177.

[21] E. Tomboulis, The 't Hooft loop in SU(2) lattice gauge theories, Phys. Rev. D23 (1981) 2371.

[22] T. G. Kovács and E. T. Tomboulis, Vortices and confinement at weak coupling, Phys. Rev. D57 (1998) 4054-4062, hep-lat/9711009.

[23] A. Alexandru and R. W. Haymaker, Vortices in $S O(3) \times Z(2)$ simulations, Phys. Rev. D62 (2000) 074509, hep-lat/0002031.

[24] T. Yoneya, Monopole condensation and quark confinement in a weak coupling SU(N) lattice gauge model, Nucl. Phys. B153 (1979) 431. 
[25] J. Ambjørn and H. Flyvbjerg, 't Hooft's nonabelian magnetic flux has zero classical energy, Phys. Lett. B97 (1980) 241.

[26] M. Lüscher, Topology of lattice gauge fields, Commun. Math. Phys. 85 (1982) 39.

[27] A. Barresi, G. Burgio and M. Müller-Preussker, SO(3) Yang-Mills theory on the lattice, hep-lat/0209011.

[28] B. A. Berg and T. Neuhaus, Multicanonical algorithms for first order phase transitions, Phys. Lett. B267 (1991) 249-253.

[29] R. C. Brower, D. A. Kessler and H. Levine, Dynamics of SU(2) lattice gauge theories, Nucl. Phys. B205 (1982) 77.

[30] I. G. Halliday and A. Schwimmer, Z(2) monopoles in lattice gauge theories, Phys. Lett. B102 (1981) 337.

[31] S. Datta and R. V. Gavai, Deconfinement transition in SO(3) gauge theory, Phys. Rev. D60 (1999) 034505, hep-lat/9901006.

[32] A. Barresi, G. Burgio and M. Müller-Preussker, Finite temperature phase transition, adjoint Polyakov loop and topology in SU(2) LGT, Nucl. Phys. Proc. Suppl. 106 (2002) 495-497, hep-lat/0110139.

[33] J. Fingberg, U. M. Heller and V. K. Mitrjushkin, Scaling in the positive plaquette model and universality in SU(2) lattice gauge theory, Nucl. Phys. B435 (1995) 311-338, hep-lat/9407011.

[34] K. Holland, P. Minkowski, M. Pepe and U. J. Wiese, Confinement without a center: the exceptional group $G(2)$, hep-lat/0209093.

[35] A. González-Arroyo, Yang-Mills fields on the 4-dimensional torus. (classical theory), hep-th/9807108.

[36] A. González-Arroyo, J. Jurkiewicz and C. P. Korthals-Altes, Ground state metamorphosis for Yang-Mills fields on a finite periodic lattice, in Proceedings of the 11th NATO Summer Institute (J. Honerkamp, et al., eds.), Plenum, 1982. 